\title{
A Cross-National Study of Subjective Sexual Well-Being Among Older Women and Men: Findings From the Global Study of Sexual Attitudes and Behaviors
}

\author{
Edward O. Laumann, Ph.D., ${ }^{1,10}$ Anthony Paik, Ph.D., ${ }^{2}$ Dale B. Glasser, Ph.D., 3 \\ Jeong-Han Kang, M.A., ${ }^{1}$ Tianfu Wang, Ph.D., ${ }^{4}$ Bernard Levinson, M.B., B.Ch., D.P.M., ${ }^{5}$ \\ Edson D. Moreira, Jr., M.D., Ph.D., 6 Alfredo Nicolosi, M.D., Ph.D., ${ }^{7,8}$ \\ and Clive Gingell, F.R.S.C. ${ }^{9}$
}

Received November 15, 2004; revision received May 5, 2005; accepted October 12, 2005 Published online: 26 April 2006

\begin{abstract}
Subjective sexual well-being refers to the cognitive and emotional evaluation of an individual's sexuality. This study examined subjective sexual well-being, explored its various aspects, examined predictors across different cultures, and investigated its possible associations with overall happiness and selected correlates, including sexual dysfunction. Data were drawn from the Global Study of Sexual Attitudes and Behaviors, a survey of 27,500 men and women aged 40-80 years in 29 countries. The cross-national variation of four aspects of sexual well-being (the emotional and physical satisfaction of sexual relationships, satisfaction with sexual health or function, and the importance of sex in one's life) was explored using cluster analysis, and relationships among sexual well-being, general happiness, and various correlates were examined using ordinary least squares regression and ordered logistic regression. Results from the cluster analysis identified three clusters: a gender-equal regime and two male-centered regimes. Despite this cultural variation, the predictors of subjective sexual well-being were found to be largely consistent across world regions.
\end{abstract}

KEY WORDS: subjective well-being; sexual function; happiness; gender roles; cross-national.

\footnotetext{
${ }^{1}$ Department of Sociology, University of Chicago, Chicago, Illinois.

${ }^{2}$ Department of Sociology, University of Iowa, Iowa City, Iowa.

${ }^{3}$ Pfizer Inc., New York, New York.

${ }^{4}$ Department of Sociology, Tsinghua University, Beijing, People's Republic of China.

${ }^{5}$ Private Practice, Johannesburg, South Africa.

${ }^{6}$ Unit of Epidemiology and Biostatistics, Oswaldo Cruz Foundation, Bahia, Brazil.

${ }^{7}$ Department of Epidemiology and Medical Informatics, National Research Council, Milan, Italy.

${ }^{8}$ GH Sergievsky Center, Columbia University School of Public Health, New York, New York.

${ }^{9}$ GSSAB Investigators' Group, Bristol Urological Institute, Southmead Hospital, Bristol, England.

${ }^{10} \mathrm{To}$ whom correspondence should be addressed at Department of Sociology, University of Chicago, 1126 E. 59th Street, Room 409, Chicago, Illinois 60637; e-mail: e-laumann@uchicago.edu.
}

\section{INTRODUCTION}

Despite increased scholarly research and public debate about the nature, the predictors, and the sociopolitical aspects of sexual problems and dysfunction (Blanker et al., 2001; Braun et al., 2000; Feldman, Goldstein, Hatzichristou, Krane, \& McKinlay, 1994; Laumann et al., 2005; Laumann, Paik, \& Rosen, 1999; Moreira, Abdo, Torres, Lobo, \& Fittipaldi, 2001; Nicolosi et al., 2004; Nicolosi, Moreira, Shirai, Bin Mohd Tambi, \& Glasser, 2003), relatively little is known about what constitutes perceived sexual health and well-being. Most people are living longer than preceding generations, and more are remaining sexually active in later life-a result of changing attitudes toward sexuality and the availability of effective therapies for sexual dysfunctions. Thus, the nature, the predictors, and the associated outcomes of sexual well-being are an increasingly important issue, 
particularly among older women and men. However, there are few studies of sexual health and well-being among individuals living in Western nations (Cain et al., 2003; Laumann, Gagnon, Michael, \& Michaels, 1994) and even less is known in non-Western and developing nations. In this research, we characterize and investigate aspects of subjective sexual well-being, defined here as the cognitive and emotional evaluation of an individual's sexuality (Oberg, Fugl-Meyer, \& Fugl-Meyer, 2002). This concept is meant to be a sexual analog to subjective well-being, which has been discussed extensively in the psychological literature and includes feelings of happiness, fulfillment, peace, and life satisfaction (Kahneman, Diener, \& Schwartz, 1999; Schwartz \& Strack, 1999).

Subjective sexual well-being refers to the perceived quality of an individual's sexuality, sexual life, and sexual relationships. We focus primarily on evaluations of sexuality in terms of satisfaction judgments, which have been central for studying the concept of overall wellbeing (Diener \& Suh, 1999; Schwartz \& Strack, 1999). There is some research on sexual satisfaction (HaavioMannila \& Kontula, 1997; Oberg et al., 2002; Waite \& Joyner, 2001); however, this research typically focuses on a single domain of sexual satisfaction in a single country. By concentrating on sexual well-being, we examined sexual satisfaction across several domains of sexuality, including satisfaction with emotional and physical aspects of relationships, satisfaction with sexual functioning, and the relative importance of sexuality in one's overall life.

In addition to characterizing the multiple domains of sexual well-being, we investigated the correlates of sexual well-being in terms of predictors and an important concomitant outcome, general happiness. Prior studies have focused on several types of predictors, including physical and psychological health, relationship characteristics, and sexual practices and attitudes. Physical health and psychological well-being have been identified as important correlates of sexual problems (Laumann et al., 1999, 2005), so we included a variety of predictors, including overall health, physical activity, smoking status, psychological distress, and sexual problems. In addition, Waite and Joyner (2001) and Haavio-Mannila and Kontula (1997) highlighted the importance of relationship characteristics and sexual practices and attitudes for sexual satisfaction. In this research, we assessed the importance of two relationship characteristics: marital status and the time horizon of the relationship. We also examined how sexual practices and sexual attitudes affect assessments of sexual well-being. Finally, we studied the strength of association between these aspects of sexual well-being and general happiness.
We investigated sexual well-being across 29 countries. National differences in sexual well-being highlight how an individual's evaluation of his or her own sexuality is embedded within a larger sociocultural context comprised of distinct normative orientations toward sexuality and their systematic organization, which we refer to as a sexual regime (Laumann et al., 1994; Widmer, Treas, \& Newcomb, 1998). Sexual regimes, as complexes of sexually relevant normative orientations, distinctively vary in the way gender differences are understood. Malecentered sexual regimes, sometimes called patriarchal regimes, emphasize the centrality of men in controlling the sexual conduct of women, while a gender-equal sexual regime is exemplified in the normative ideal of the companionate marriage, which emphasizes equality between intimate partners (Blumstein \& Schwartz, 1983; Reiss, 1967; Reiss \& Reiss, 1997; Waite \& Joyner, 2001). The roots of these differing normative traditions are deep and far ranging, derived from fundamental, cultural differences across societies in family and religious traditions and historical experiences. Because of their companionate orientation, gender-equal sexual regimes, we hypothesize, will be associated with higher levels of sexual well-being than male-centered regimes. We also expected that gender differences in sexual well-being will be lower in the former in comparison with the latter.

The Global Study of Sexual Attitudes and Beliefs (GSSAB) is the first large, multi-country survey that systematically studied attitudes, beliefs, and health in sexual relationships in middle-aged and older adults (Laumann et al., 2005; Nicolosi et al., 2004). In this article, we used the GSSAB data to address the following issues: (1) to provide an empirical measurement of aspects of sexual well-being; (2) to address crosscultural variation of sexual well-being in terms of how cultures vary with respect to sexual regimes and gender roles; (3) to assess the importance of physical and psychological health, relationship characteristics, and sexual attitudes and beliefs as covariates of various aspects of sexual well-being; and (4) to assess the overall connection between sexual well-being and overall happiness.

\section{METHOD}

\section{Participants}

The survey involved 13,882 women and 13,618 men, aged 40-80 years, in 29 countries, representing many world regions. The data were collected using standard sampling methods in each country. 
Random-digit dialing was used in Europe, Israel, North America, Brazil, Australia, and New Zealand. These respondents were randomly selected within households by asking for the man or woman aged 40-80 years who had had the most recent birthday, and the interviews were conducted by telephone. Because of a bias against telephone interviews among the lower income groups, a mix of telephone and in-person interviews was used in Mexico. In Japan, a mail survey was used. Names were randomly selected from the national telephone database, and those people were mailed a questionnaire with a postage-paid return envelope and an incentive check of U.S. \$4.

Alternative protocols were employed in countries with low telephone connection rates. In the Middle East and South Africa, a door-to-door protocol was used. Homes were selected using a random starting point, and interviewers contacted every third house. Respondents self-completed a questionnaire and returned it to the interviewer. In Asian countries, other than Japan, we used an intercept method (rural areas were not included because of logistical problems). Individuals were randomly contacted in public areas by an interviewer who asked them to self-complete a questionnaire. Regardless of sampling method, the respondents were contacted by same-gender interviewers (not applicable in Japan).

To ensure accuracy of translation of the English version of the interview instrument into the various languages spoken in the countries we studied, the country-based polling organization contracted to conduct the survey in that country had its bilingual staff translate the questionnaire into the local language, under the supervision of a bilingual, seniorphysician consultant who had a clinical practice in that country.

Overall, 192,504 individuals were contacted, of whom 45,930 were not eligible to participate. Of the 146,574 eligible individuals, 94,672 refused to participate at the introduction, and 24,388 individuals interrupted the interview. A total of 27,514 individuals completed the questionnaire, for a response rate of $19 \%(29.7 \%$ in countries in which the sampling involved direct personal contact, $15.3 \%$ in the countries in which the interview was administered by telephone, and 33.3\% in Japan). The high attrition rate was, in part, attributable to the protocol which stipulated that, in the interest of preserving respondents' anonymity, no call backs were permitted to find better times for interviews or to try to persuade "refusers" to participate. We assumed that the main reason for refusal was a generic unavailability to participate in a survey $(67 \%$ of the contacts refused participation at introduction, and if they were excluded, the response rate would be 57\%). Fourteen individuals in the U.S. sample refused to answer an age question, and the final sample size was 27,500. ${ }^{11}$

Verbal consent was obtained from all study participants. They were also informed about the following issues: (1) all information obtained would be used in aggregate, (2) responses were voluntary, (3) the confidentiality and the privacy of their responses were protected because no personal identifiers were coded into the interview instruments, (4) no list of respondents was retained, and (5) "refusers" were not called back in an effort to convert them to participating respondents.

The GSSAB questionnaire took an average of about 20 min to complete. It asked for information about demographic details, health, general satisfaction, relationships, sexual behavior and practices, and attitudes and beliefs regarding sexuality. Both sexually active and inactive persons were included in the analysis, unless specifically noted. Some questions, for example, those pertaining to emotional and physical satisfaction with one's sexual partner in the past year, were not asked of respondents who reported not having a sexual partner in the past year. On the other hand, everyone was asked about the importance of sexuality in one's life and satisfaction with sexual functioning.

\section{Dependent Measures}

\section{Subjective Sexual Well-Being}

To assess subjective sexual well-being, four measures were employed to tap various evaluative domains of sexuality. Two questions asked respondents about their emotional and physical satisfaction with their sexual relationships. Respondents were first asked: "During the past 12 months, how physically pleasurable did you find your relationship with your partner to be?" Similarly, respondents were asked: "During the past 12 months, how emotionally satisfying did you find your relationship with your partner to be?" Response options for both questions were on a 5-point scale, ranging from "not at all satisfying" to "extremely satisfying." In some of the following analyses, we combined emotional and physical satisfaction to reflect "relational satisfaction" because the two were highly intercorrelated $(\alpha=.80)$. Satisfaction

\footnotetext{
${ }^{11}$ The 500 cases collected in Hong Kong were dropped from the analyses reported here because they were too few to sustain statistical analysis and there was no statistical justification for assigning them to the sample collected in mainland China. As a result, the analyses reported here were based on a total sample of 27,000 .
} 


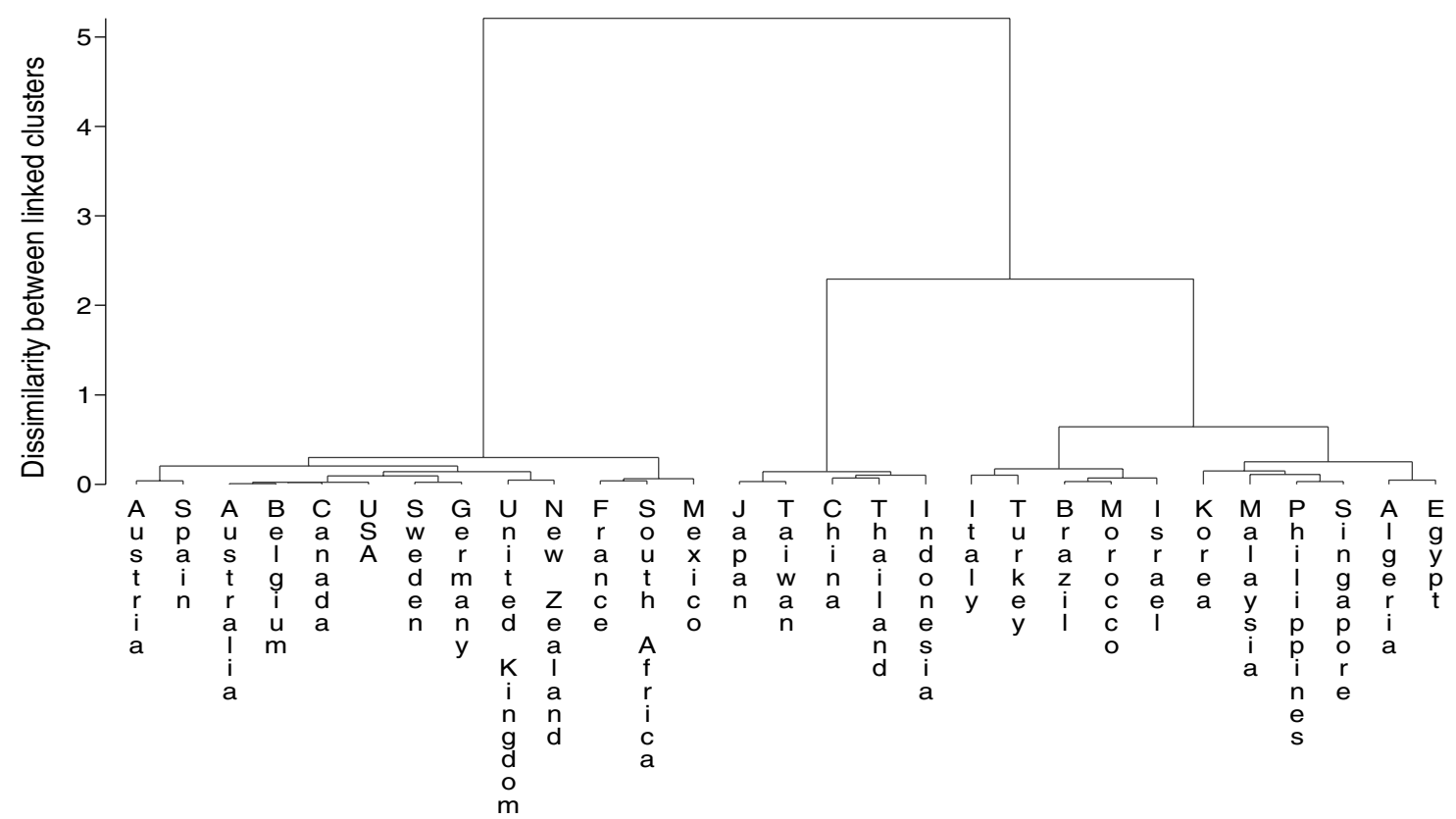

Fig. 1. Dendrogram depicting the results of cluster analysis, assigning countries to clusters based on positive responses to four aspects of subjective sexual well-being.

with sexual function was assessed with the following question: "If you were to spend the rest of your life with your sexual function/sexual health the way it is today, how would you feel about this?" Response options were on a 5-point scale, ranging from "very dissatisfied" to "very satisfied". And finally, respondents were asked: "How important a part of your overall life would you say that sex is. ..?" Response options ranged from "not important at all" to "extremely important." While this last question did not refer to the past 12 months, it followed a series of three questions that specifically referred to the past 12 months. The immediately preceding question asked how often the respondent had thought about sex in the past month. Thus, the context of the question about the importance of sex in one's life was clearly referring to an assessment of one's current view of the matter.

\section{General Happiness}

With respect to the major concomitant outcome, general happiness, respondents were asked (before they were asked any of the questions about sexual well-being): "Generally, how happy have you been with your life as a whole [physical, social, family, work] during the past 12 months?" Response categories ranged from "not at all happy" to "extremely happy."

\section{Independent Measures}

\section{Physical and Psychological Health}

Self-rated health was asked in a 4-point scale, ranging from "poor" to "excellent." The level of physical activity and exercise was asked in a 3-point scale, and introduced in the analysis by setting "above average" as a reference category. Respondents were also asked if they had ever been diagnosed with various health conditions. A variable for vascular conditions was measured by counting the number of diagnoses of hypertension, diabetes, heart disease, high cholesterol, and stroke. Diagnosed with depression or diagnosed with prostate disease for men were introduced separately in the analysis with dummy variables. Sexual dysfunctions, such as lacked interest in having sex, physical pain during intercourse, erectile difficulties for men, and lubrication problems for women, indicated if respondents experienced these conditions for at least 2 months during the last 12 months.

\section{Relationship Characteristics}

Respondents were asked if they currently have more than one sexual partner. Marital status consisted of three categories: being married, cohabiting, and living alone. The last category, living alone, implied dating rather 
than lack of a sexual partner because regressions of sexual well-being or general happiness were restricted to sexually active respondents. A variable for expected time horizon with partner also consisted of three categories: believe to be together forever, hope to remain together, and worry/doubt about staying together. This variable, as well as the following variables for sexual practice, was defined only for sexually active respondents for the past 12 months.

\section{Sexual Practices}

The average frequency of sex over the last 12 months was recoded into three categories: less than monthly, two or three times per month, and at least weekly. The average amount of time spent on foreplay was coded into a 5-point scale: zero minutes, less than $5 \mathrm{~min}, 6-15 \mathrm{~min}, 16-30 \mathrm{~min}$, or more than $30 \mathrm{~min}$.

\section{Sexual Attitudes}

Five items for sexual attitudes related to aging or gender were employed. Response categories for each item ranged from "strongly disagree" to "strongly agree." The five items were: "Older people no longer want sex," "Men can enjoy sex even with partners that they don't love," "A "real man" is ready for sex at any time," "Women have greater control over sexual desires than men," and "Women have a duty to meet their partner's sexual needs."

\section{Statistical Analyses}

A cluster analysis was employed to examine national differences in sexual well-being among sexually active and inactive respondents whenever appropriate. To perform this analysis, the responses of the 19,956 sexually active men and women (i.e., respondents having intercourse in the past year) from the 29 countries were aggregated into a 29-by-4 matrix of percentages for the two questions referring to physical and emotional satisfaction with one's sex partner in the past year. This was joined to another 29-by-4 matrix of percentages that included both sexually active and inactive respondents' answers to the two questions on the importance of sexuality and satisfaction with sexual function. The 29 rows in the matrix represented 29 countries. The eight columns in the matrix reflected the four aspects of sexual well-being for men and women, respectively. The top two categories for each aspect were collapsed to identify positive answers as follows: (1) being extremely or very
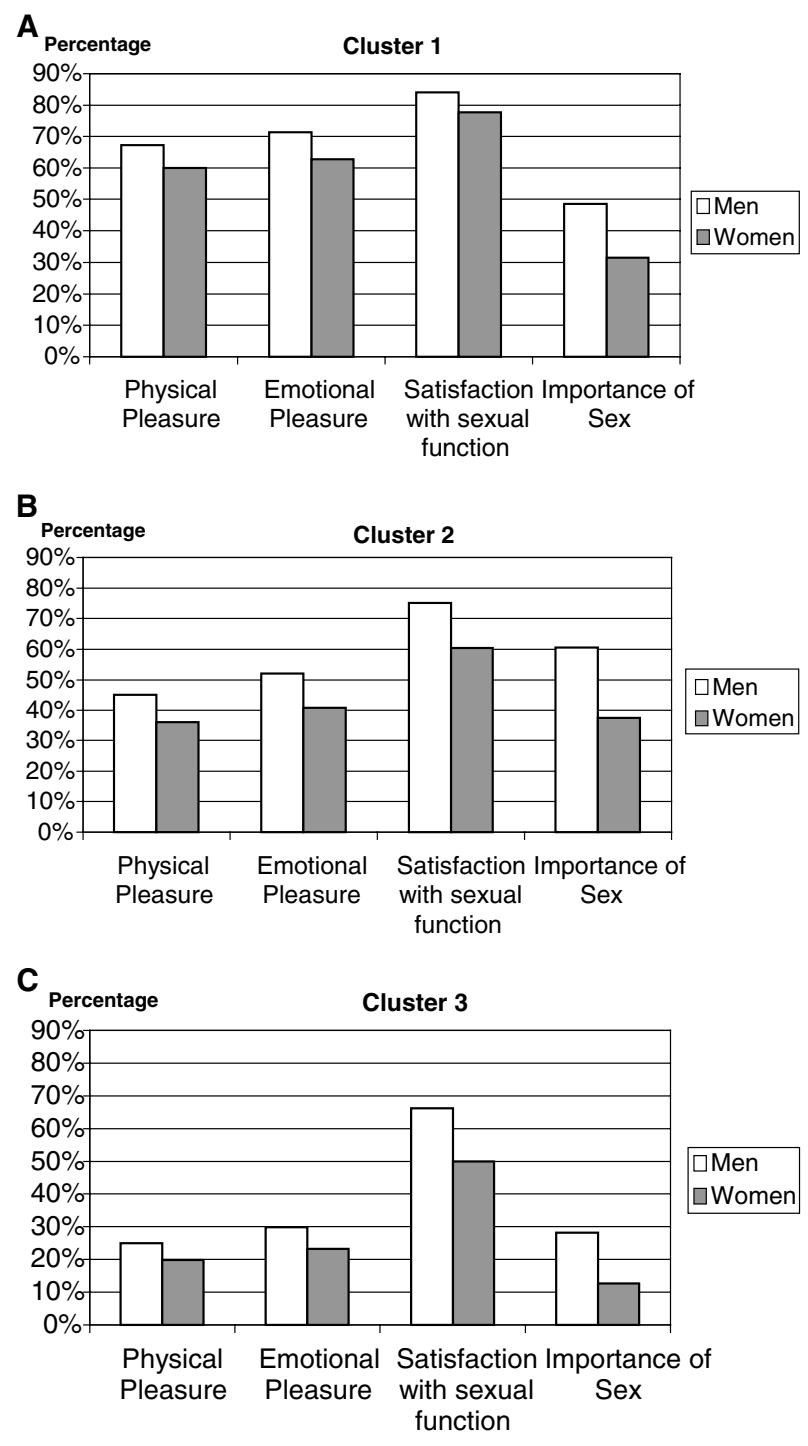

Fig. 2. Four aspects of subjective sexual well-being by gender (panel A: cluster 1; panel B: cluster 2; panel C: cluster 3).

satisfied with the physical and (2) with the emotional aspects of their relationships; (3) being very or somewhat satisfied with their level of sexual function; and (4) finding that sex was an extremely or very important part of their overall life.

Applied to the study of national differences, the cluster analysis investigated the extent to which sexual well-being was a country-specific phenomenon or a consequence of larger cultural patterns, such as sexual regimes. Cluster analysis has been previously employed to analyze patterns of normative orientations within the United States as well as to identify countries that displayed shared sexual regimes (Laumann et al., 1994; 


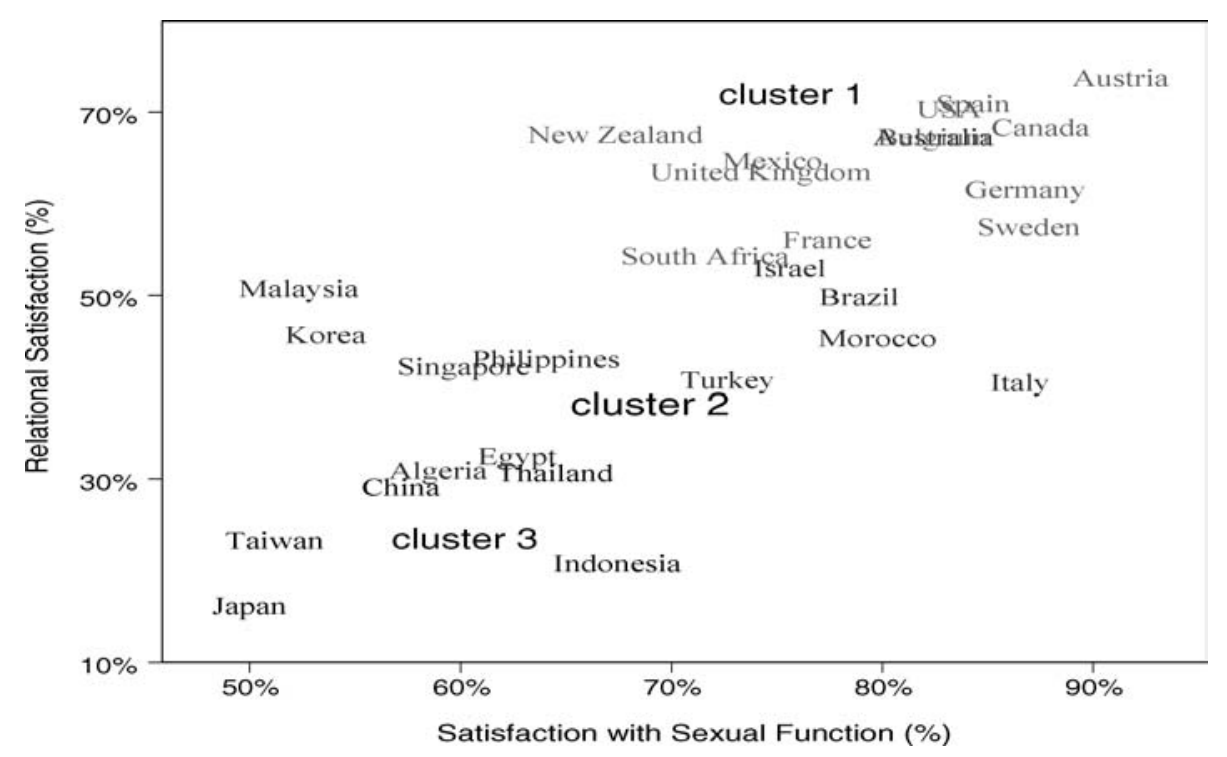

Fig. 3. Relational satisfaction vs. satisfaction with sexual function (scatter plot with countries as points).

Widmer et al., 1998). We employed Ward's (1963) linkage hierarchical clustering analysis. While researchers have considerable latitude in determining the number of clusters identified, a criterion of model fit is to examine how splitting clusters reduces the amount of withincluster variance. Since the matrix was developed with men and women separately, identified clusters reflect not only between country differences but also gender differences.

To identify predictors of subjective sexual well-being at the individual level, we used the results of the cluster analysis-the number of clusters and the assignment of countries to a specific cluster-to group countries in the remaining analyses. We first conducted factor analyses to identify latent constructs for the multiple aspects of subjective well-being and used these results to specify dependent measures. We then conducted multiple regression analysis on aspects of subjective sexual wellbeing by cluster and gender. The first dependent variable was relationship satisfaction, which was the average of the highly correlated indicators of physical and emotional satisfaction $(\alpha=.80)$. The remaining two were satisfaction with sexual function and the importance of sex. Ordered logistic regressions were calculated for the last two, which were 5-point scales. Lastly, we conducted an ordered logistic regression of overall happiness on the four aspects of sexual well-being with health and life-course factors. All of these regressions were limited to sexually active respondents because some of the independent variables (e.g., frequency of sex in the past year and time spent in foreplay) were not asked of sexually inactive persons.

\section{RESULTS}

\section{Cluster Analysis Using Four Aspects of Sexual Well-being}

Figure 1 presents a dendrogram depicting the results of the cluster analysis, which used percent distributions of positive responses to the four aspects of subjective well-being by country and gender as its input. The cluster analysis assigned countries to particular clusters based on having similar profiles in terms of positive responses to subjective well-being with other countries in the cluster and dissimilar profiles to countries assigned to the other clusters. The three-cluster solution reduced a considerable amount of the observed dissimilarity among countries in a given cluster and appeared to provide a parsimonious result as compared with a four- or higher-cluster solution, which resulted in an additional splitting of the clusters but did not show much dissimilarity between the additional clusters.

Table I presents the percent distributions of positive responses to the four aspects of subjective sexual wellbeing by country and gender for each analysis. The first cluster of countries consisted of Western European nations (Austria, Belgium, France, Germany, Spain, Sweden, and the United Kingdom), Mexico, and nonEuropean, English-speaking nations, including Australia, Canada, New Zealand, South Africa, and the United States. The second cluster was constituted primarily by Mediterranean (Algeria, Egypt, Israel, Italy, Morocco, and Turkey) and Asian countries (Korea, Malaysia, and the 
Table I. Positive Responses to Four Aspects of Subjective Sexual Well-Being by Country and by Gender, Arranged by Cluster Membership: Percentage Distributions

\begin{tabular}{|c|c|c|c|c|c|c|c|c|}
\hline \multirow[b]{2}{*}{ Country } & \multicolumn{2}{|c|}{ Physical pleasure $^{a}$} & \multicolumn{2}{|c|}{ Emotional pleasure $^{b}$} & \multicolumn{2}{|c|}{$\begin{array}{l}\text { Satisfaction with sexual } \\
\text { function }\end{array}$} & \multicolumn{2}{|c|}{$\begin{array}{l}\text { Importance of } \\
\operatorname{sex}^{d}\end{array}$} \\
\hline & Men & Women & Men & Women & Men & Women & Men & Women \\
\hline \multicolumn{9}{|c|}{ Gender-equal sexual regime } \\
\hline \multicolumn{9}{|c|}{$\begin{array}{l}\text { 1. Western European and European-Linked Western Countries (high levels of sexual satisfaction). } \\
n=14,503 \text { (male }=7,224 ; \text { female }=7,279)\end{array}$} \\
\hline Australia & 67.7 & 62.9 & 72.3 & 68.0 & 82.1 & 82.7 & 43.6 & 29.7 \\
\hline Austria & 79.7 & 63.0 & 83.4 & 70.6 & 91.4 & 91.1 & 56.3 & 35.5 \\
\hline Belgium & 66.2 & 65.1 & 72.3 & 67.3 & 86.7 & 78.1 & 48.5 & 32.3 \\
\hline Canada & 71.2 & 67.8 & 73.7 & 62.5 & 87.4 & 87.6 & 44.6 & 33.8 \\
\hline France & 61.2 & 51.7 & 59.9 & 53.2 & 85.2 & 69.6 & 55.8 & 39.4 \\
\hline Mexico & 69.1 & 57.4 & 71.8 & 62.3 & 85.2 & 64.4 & 59.0 & 36.6 \\
\hline New Zealand & 71.0 & 62.5 & 76.9 & 61.6 & 70.6 & 64.1 & 35.7 & 21.6 \\
\hline South Africa & 60.6 & 46.9 & 64.7 & 46.7 & 77.7 & 65.5 & 54.5 & 26.3 \\
\hline Spain & 72.9 & 67.5 & 76.0 & 69.1 & 90.2 & 78.4 & 64.1 & 33.8 \\
\hline Sweden & 58.2 & 55.9 & 57.8 & 59.7 & 89.4 & 84.5 & 46.4 & 31.7 \\
\hline Germany & 62.5 & 56.8 & 69.0 & 59.9 & 87.7 & 85.9 & 41.1 & 33.1 \\
\hline United Kingdom & 60.9 & 56.0 & 71.3 & 67.5 & 73.9 & 74.6 & 44.8 & 29.6 \\
\hline USA & 72.9 & 65.0 & 77.1 & 68.0 & 83.9 & 82.4 & 36.6 & 27.7 \\
\hline Mean & 67.3 & 59.9 & 71.2 & 62.8 & 83.9 & 77.6 & 48.6 & 31.6 \\
\hline \multirow{2}{*}{\multicolumn{9}{|c|}{$\begin{array}{l}\text { Male-centered sexual regimes } \\
\text { 2. Islamic and selected Asian and European countries (middle levels of sexual satisfaction). } \\
n=8,997 \text { (male }=4,394 ; \text { female }=4,603)\end{array}$}} \\
\hline & & & & & & & & \\
\hline Algeria & 21.1 & 33.7 & 46.3 & 24.6 & 70.2 & 47.6 & 53.9 & 33.8 \\
\hline Brazil & 59.3 & 39.7 & 60.8 & 41.3 & 88.2 & 69.6 & 74.6 & 43.1 \\
\hline Egypt & 36.2 & 23.6 & 44.4 & 27.6 & 73.2 & 52.2 & 52.8 & 40.4 \\
\hline Israel & 51.8 & 47.7 & 59.3 & 54.8 & 76.0 & 75.1 & 64.2 & 53.6 \\
\hline Italy & 42.8 & 31.6 & 48.8 & 40.6 & 90.9 & 82.1 & 58.0 & 32.7 \\
\hline Korea & 47.3 & 36.2 & 54.0 & 47.2 & 60.9 & 46.3 & 73.1 & 46.4 \\
\hline Malaysia & 45.6 & 50.2 & 50.9 & 58.0 & 60.1 & 44.5 & 45.8 & 34.9 \\
\hline Morocco & 47.4 & 37.0 & 53.7 & 45.0 & 82.2 & 77.3 & 73.3 & 42.6 \\
\hline Philippines & 46.5 & 40.2 & 48.7 & 38.5 & 71.3 & 56.8 & 56.9 & 30.2 \\
\hline Singapore & 46.8 & 34.0 & 50.6 & 39.2 & 65.1 & 55.1 & 43.4 & 25.9 \\
\hline Turkey & 50.9 & 25.4 & 55.6 & 33.0 & 88.6 & 56.7 & 69.5 & 28.6 \\
\hline Mean & 45.1 & 36.3 & 52.1 & 40.9 & 75.2 & 60.3 & 60.5 & 37.5 \\
\hline \multicolumn{9}{|c|}{ 3. East Asian countries (low levels of sexual satisfaction). $n=3,500($ male $=1,750 ;$ female $=1,750)$} \\
\hline China & 25.2 & 24.4 & 36.0 & 32.8 & 68.7 & 45.5 & 28.8 & 17.5 \\
\hline Indonesia & 22.0 & 24.7 & 18.5 & 19.9 & 73.8 & 61.0 & 35.4 & 16.6 \\
\hline Japan & 17.6 & 9.8 & 23.6 & 15.5 & 60.3 & 39.7 & 27.8 & 12.0 \\
\hline Taiwan & 21.5 & 19.4 & 28.7 & 25.4 & 60.0 & 42.3 & 24.7 & 7.3 \\
\hline Thailand & 38.1 & 21.0 & 42.6 & 22.8 & 67.6 & 61.3 & 23.8 & 10.1 \\
\hline Mean & 24.9 & 19.8 & 29.9 & 23.3 & 66.1 & 50.0 & 28.1 & 12.7 \\
\hline
\end{tabular}

${ }^{a}$ Extremely/very pleasurable as opposed to moderately/slightly/not at all pleasurable.

${ }^{b}$ Extremely/very satisfying as opposed to moderately/slightly/not at all satisfying.

${ }^{c}$ Very/somewhat satisfied as opposed to neither satisfied nor dissatisfied or somewhat/very dissatisfied.

${ }^{d}$ Extremely/very important as opposed to moderately/slightly/not at all important.

Philippines), but also included Brazil. The third cluster included only Asian countries, such as China, Indonesia, Japan, Taiwan, and Thailand.

To facilitate interpretation of this clustering, Fig. 2a-c present bar charts of the mean positive responses to the four aspects of subjective sexual well-being by gender and by cluster. Two general results were readily apparent. First, with respect to all four aspects of subjective sexual well-being, men consistently had higher means than women in all three clusters. For example, $71 \%$ of men in comparison to $63 \%$ of women in Cluster 1 were extremely or very satisfied emotionally with their 
Table II. Ordinary Least Squares Regressions on Relational Satisfaction and Ordered Logistic Regressions on Satisfaction With Sexual Functioning and the Importance of Sex by Gender and Cluster.

\begin{tabular}{|c|c|c|c|c|c|c|}
\hline \multirow[b]{3}{*}{ Variables } & \multicolumn{6}{|c|}{ Satisfaction with sexual functioning } \\
\hline & \multicolumn{2}{|c|}{ Cluster 1} & \multicolumn{2}{|c|}{ Cluster 2} & \multicolumn{2}{|c|}{ Cluster 3} \\
\hline & Men & Women & Men & Women & Men & Women \\
\hline \multicolumn{7}{|c|}{$\begin{array}{l}\text { A. Regression of relational (physical and emotional) satisfaction } \\
\text { Demographic controls }\end{array}$} \\
\hline Age & -.000 & -.001 & $-.009^{\dagger}$ & -.001 & $-.006^{*}$ & .002 \\
\hline \multicolumn{7}{|l|}{ Education (high school) } \\
\hline Less than high school & -.041 & .018 & -.053 & $-.203^{\dagger}$ & -.001 & -.042 \\
\hline Higher than high school & -.029 & -.036 & $.076^{*}$ & -.014 & .040 & -.002 \\
\hline \multicolumn{7}{|l|}{ Employment status (working) } \\
\hline Homemaker & .131 & $.079^{*}$ & .119 & .033 & .224 & -.026 \\
\hline Retired & -.014 & -.002 & -.015 & -.072 & .076 & .054 \\
\hline Unemployed & .069 & -.011 & .095 & $-.295^{\dagger}$ & -.142 & -.112 \\
\hline \multicolumn{7}{|l|}{ Physical health } \\
\hline Health (self-estimated, 4-points) & $.128^{\dagger}$ & $.136^{\dagger}$ & $.228^{\dagger}$ & $.264^{\dagger}$ & $.207^{\dagger}$ & $.233^{\dagger}$ \\
\hline \multicolumn{7}{|l|}{ Physical activity (high) } \\
\hline Average & -.012 & -.042 & $-.135^{\dagger}$ & .038 & -.089 & $-.139^{*}$ \\
\hline Low & -.005 & -.053 & $-.230^{\dagger}$ & -.010 & $-.144^{*}$ & -.030 \\
\hline \multicolumn{7}{|l|}{ Vascular condition (none) } \\
\hline One & -.008 & .033 & .015 & .055 & $.091^{*}$ & $-.130^{*}$ \\
\hline Two or more & $-.076^{*}$ & .045 & $.093^{*}$ & .036 & .032 & .133 \\
\hline Diagnosed w/prostate disease & -.050 & .000 & .029 & .000 & .114 & .000 \\
\hline Currently smoking & .015 & .001 & .007 & .040 & $-.078^{*}$ & .045 \\
\hline \multicolumn{7}{|l|}{ Psychological health } \\
\hline Diagnosed w/depression & $-.103^{\dagger}$ & -.058 & $-.154^{\dagger}$ & $-.125^{\dagger}$ & .034 & $-.448^{\dagger}$ \\
\hline Loss of job in past 3 years & -.028 & -.056 & -.035 & .038 & -.031 & .047 \\
\hline Divorced in past 3 years & $.257^{\dagger}$ & .139 & .012 & .025 & .002 & -.229 \\
\hline Serious financial/work "setback" in past 3 years & $-.092^{\dagger}$ & $-.189^{\dagger}$ & -.063 & $-.092^{*}$ & .062 & -.019 \\
\hline Serious medical condition in past 3 years & $.082^{*}$ & -.037 & .053 & .027 & .034 & .087 \\
\hline \multicolumn{7}{|l|}{ Sexual dysfunctions } \\
\hline Lacked interest in sex past year & -.055 & $-.145^{\dagger}$ & $-.095^{*}$ & $-.118^{\dagger}$ & -.025 & $-.185^{\dagger}$ \\
\hline Experienced physical pain during sex past year & -.118 & -.071 & -.068 & -.077 & -.022 & -.043 \\
\hline Trouble achieving/maintaining erection past year & $-.107^{\dagger}$ & & .029 & & -.008 & \\
\hline Trouble becoming adequately lubricated past year & & -.001 & & .005 & & $-.127^{*}$ \\
\hline \multicolumn{7}{|l|}{ Relationship characteristics and sexual practices } \\
\hline \multicolumn{7}{|l|}{ Marital status (married) } \\
\hline Cohabiting & .004 & $.147^{\dagger}$ & $.165^{*}$ & .040 & .053 & .253 \\
\hline Dating & $.142^{\dagger}$ & $.193^{\dagger}$ & $.168^{*}$ & .103 & $.389^{\dagger}$ & .085 \\
\hline \multicolumn{7}{|l|}{ Time horizon (together forever) } \\
\hline Hope to stay together & $-.268^{\dagger}$ & $-.296^{\dagger}$ & $-.078^{\dagger}$ & -.030 & $-.151^{\dagger}$ & $-.190^{\dagger}$ \\
\hline Less likely to stay together & $-.552^{\dagger}$ & $-.701^{\dagger}$ & $-.332^{\dagger}$ & $-.286^{\dagger}$ & $-.449^{\dagger}$ & $-.481^{\dagger}$ \\
\hline Multiple sex partners & $-.210^{\dagger}$ & -.136 & $-.128^{\dagger}$ & -.064 & .044 & -.044 \\
\hline \multicolumn{7}{|l|}{ Frequency of sex (less than monthly) } \\
\hline Two or three times per month & $.273^{\dagger}$ & $.171^{\dagger}$ & $.164^{\dagger}$ & .022 & $.237^{\dagger}$ & $.154^{\dagger}$ \\
\hline Weekly or more & $.395^{\dagger}$ & $.274^{\dagger}$ & $.230^{\dagger}$ & $.139^{\dagger}$ & $.394^{\dagger}$ & $.388^{\dagger}$ \\
\hline Foreplay time & $.081^{\dagger}$ & $.113^{\dagger}$ & $.052^{\dagger}$ & $.124^{\dagger}$ & $.072^{\dagger}$ & $.106^{\dagger}$ \\
\hline \multicolumn{7}{|l|}{ Real men sexual attitudes } \\
\hline Older people no longer want sex & -.005 & $-.051^{\dagger}$ & $.028^{*}$ & -.009 & -.010 & -.038 \\
\hline Men can enjoy sex even without love & $-.017^{*}$ & $-.037^{\dagger}$ & $-.025^{*}$ & $-.026^{*}$ & $-.041^{*}$ & $-.049^{*}$ \\
\hline Real men' is ready for sex at any time & $-.023^{\dagger}$ & $-.024^{\dagger}$ & .014 & $.025^{*}$ & .014 & .029 \\
\hline Women have greater control over sexual desires & .011 & -.012 & .013 & -.006 & $.058^{*}$ & .035 \\
\hline Women have a duty to meet partner's sexual needs & $-.020^{*}$ & .007 & $-.035^{\dagger}$ & .020 & -.010 & .028 \\
\hline Constant & $3.352^{\dagger}$ & $3.568^{\dagger}$ & $3.335^{\dagger}$ & $2.601^{\dagger}$ & $2.659^{\dagger}$ & $2.243^{\dagger}$ \\
\hline$n$ & 4609 & 3907 & 3181 & 2689 & 1364 & 1078 \\
\hline$R^{2}$ & .16 & .21 & .17 & .22 & .20 & .29 \\
\hline
\end{tabular}


Table II. Continued

\begin{tabular}{|c|c|c|c|c|c|c|}
\hline \multirow[b]{3}{*}{ Variables } & \multicolumn{6}{|c|}{ Satisfaction with sexual functioning } \\
\hline & \multicolumn{2}{|c|}{ Cluster 1} & \multicolumn{2}{|c|}{ Cluster 2} & \multicolumn{2}{|c|}{ Cluster 3} \\
\hline & Men & Women & Men & Women & Men & Women \\
\hline \multicolumn{7}{|l|}{ B. Ordered logit of satisfaction with sexual function } \\
\hline Age & $-.015^{\dagger}$ & -.004 & -.001 & $.011^{*}$ & -.011 & -.001 \\
\hline \multicolumn{7}{|l|}{ Education (high school) } \\
\hline Less than high school & .088 & .063 & $.333^{\dagger}$ & -.071 & $-.325^{*}$ & .111 \\
\hline Higher than high school & -.036 & -.004 & $.262^{\dagger}$ & .032 & -.098 & .229 \\
\hline \multicolumn{7}{|l|}{ Employment status (working) } \\
\hline Homemaker & -.118 & -.037 & -.249 & -.047 & .128 & .105 \\
\hline Retired & -.080 & .140 & -.048 & -.093 & $.338^{*}$ & .068 \\
\hline Unemployed & -.122 & .208 & -.233 & $-.380^{*}$ & -.285 & .425 \\
\hline \multicolumn{7}{|l|}{ Physical health } \\
\hline Health (self-estimated, 4-points) & $.229^{\dagger}$ & $.241^{\dagger}$ & $.353^{\dagger}$ & $.444^{\dagger}$ & $.205^{\dagger}$ & .077 \\
\hline \multicolumn{7}{|l|}{ Physical activity (high) } \\
\hline Average & $-.215^{\dagger}$ & $-.207^{*}$ & $-.380^{\dagger}$ & .020 & -.044 & -.231 \\
\hline Low & $-.272^{\dagger}$ & $-.335^{\dagger}$ & $-.501^{\dagger}$ & -.074 & -.177 & -.290 \\
\hline \multicolumn{7}{|l|}{ Vascular condition (none) } \\
\hline One & -.025 & -.014 & .131 & $.355^{\dagger}$ & .177 & -.263 \\
\hline Two or more & -.099 & .052 & -.008 & $.365^{\dagger}$ & .065 & -.033 \\
\hline Diagnosed w/prostate disease & -.124 & & -.123 & & $.465^{*}$ & \\
\hline Currently smoking & .030 & .154 & -.101 & $.212^{*}$ & $-.305^{\dagger}$ & .104 \\
\hline \multicolumn{7}{|l|}{ Psychological health } \\
\hline Diagnosed w/depression & -.084 & -.172 & -.158 & .040 & -.120 & -.082 \\
\hline Loss of job in past 3 years & -.049 & -.092 & -.103 & -.261 & -.245 & -.420 \\
\hline Divorced in past 3 years & .233 & .176 & .302 & $-.695^{\dagger}$ & .280 & -.038 \\
\hline Serious financial/work 'setback' in past 3 years & $-.265^{*}$ & $-.372^{\dagger}$ & -.020 & .042 & -.009 & $.563^{\dagger}$ \\
\hline Serious medical condition in past 3 years & .053 & -.081 & .135 & .127 & -.190 & .285 \\
\hline \multicolumn{7}{|l|}{ Sexual dysfunctions } \\
\hline Lacked interest in sex past year & $-.247^{\dagger}$ & $-.617^{\dagger}$ & $-.526^{\dagger}$ & $-.271^{\dagger}$ & $-.783^{\dagger}$ & $-.301^{*}$ \\
\hline Experienced physical pain during sex past year & -.215 & $-.553^{\dagger}$ & .073 & $-.368^{\dagger}$ & .236 & $-.320^{*}$ \\
\hline Trouble achieving/maintaining erection past year & $-.800^{\dagger}$ & & $-.486^{\dagger}$ & & -.151 & \\
\hline Trouble becoming adequately lubricated past year & & $-.287^{\dagger}$ & & -.121 & & -.162 \\
\hline \multicolumn{7}{|l|}{ Relationship characteristics and sexual practices } \\
\hline \multicolumn{7}{|l|}{ Marital status (married) } \\
\hline Cohabiting & $.346^{\dagger}$ & $.328^{*}$ & $.528^{*}$ & .480 & -.329 & -.491 \\
\hline Dating & $.411^{\dagger}$ & .237 & .156 & $.799^{\dagger}$ & .277 & .438 \\
\hline \multicolumn{7}{|l|}{ Time horizon (together forever) } \\
\hline Hope to stay together & $-.355^{\dagger}$ & $-.471^{\dagger}$ & $-.431^{\dagger}$ & $-.304^{\dagger}$ & .139 & -.100 \\
\hline Less likely to stay together & $-.649^{\dagger}$ & $-.790^{\dagger}$ & $-.992^{\dagger}$ & $-1.043^{\dagger}$ & .053 & $-.941^{\dagger}$ \\
\hline Multiple sex partners & -.028 & -.177 & $.415^{\dagger}$ & .123 & .106 & .160 \\
\hline \multicolumn{7}{|l|}{ Frequency of sex (less than monthly) } \\
\hline Two or three times per month & $.240^{*}$ & $.357^{\dagger}$ & $.718^{\dagger}$ & $.465^{\dagger}$ & $.279^{*}$ & $.385^{\dagger}$ \\
\hline Weekly or more & $.730^{\dagger}$ & $.787^{\dagger}$ & $1.271^{\dagger}$ & $.841^{\dagger}$ & $.749^{\dagger}$ & $.891^{\dagger}$ \\
\hline Foreplay time & $.167^{\dagger}$ & $.268^{\dagger}$ & $.218^{\dagger}$ & $.446^{\dagger}$ & $.225^{\dagger}$ & $.331^{\dagger}$ \\
\hline Real men sexual attitudes & & & & & & \\
\hline Older people no longer want sex & $-.142^{\dagger}$ & $-.167^{\dagger}$ & $-.064^{*}$ & $-.136^{\dagger}$ & $-.276^{\dagger}$ & -.112 \\
\hline Men can enjoy sex even without love & .009 & .023 & $.080^{\dagger}$ & .041 & -.059 & -.063 \\
\hline Real men' is ready for sex at any time & .005 & -.008 & .038 & .014 & $.194^{\dagger}$ & .088 \\
\hline Women have greater control over sexual desires & $.090^{\dagger}$ & -.046 & $.127^{\dagger}$ & $.129^{\dagger}$ & .118 & .123 \\
\hline Women have a duty to meet partner's sexual needs & .007 & -.002 & -.034 & -.024 & .083 & .022 \\
\hline Constant & & & & & & \\
\hline$n$ & 4612 & 3913 & 3188 & 2689 & 1364 & 1078 \\
\hline$L^{2}$ & 592.64 & 692.47 & 760.2 & 687.64 & 199.19 & 176.21 \\
\hline C. Ordered Logit of importance of sex in life & & & & & & \\
\hline Demographic controls & & & & & & \\
\hline Age & $-.008^{*}$ & $-.021^{\dagger}$ & $-.011^{*}$ & $-.021^{\dagger}$ & -.013 & -.003 \\
\hline
\end{tabular}


Table II. Continued

\begin{tabular}{|c|c|c|c|c|c|c|}
\hline \multirow[b]{3}{*}{ Variables } & \multicolumn{6}{|c|}{ Satisfaction with sexual functioning } \\
\hline & \multicolumn{2}{|c|}{ Cluster 1} & \multicolumn{2}{|c|}{ Cluster 2} & \multicolumn{2}{|c|}{ Cluster 3} \\
\hline & Men & Women & Men & Women & Men & Women \\
\hline \multicolumn{7}{|l|}{ Education (high school) } \\
\hline Less than high school & .121 & -.152 & .123 & $-.278^{\dagger}$ & -.217 & .132 \\
\hline Higher than high school & -.009 & .032 & .127 & .076 & -.057 & $.396^{*}$ \\
\hline \multicolumn{7}{|l|}{ Employment status (working) } \\
\hline Homemaker & .077 & -.105 & -.177 & .052 & .193 & .068 \\
\hline Retired & $-.260^{\dagger}$ & -.162 & -.074 & -.276 & .238 & $-.450^{*}$ \\
\hline Unemployed & -.229 & -.013 & .104 & .127 & .088 & -.278 \\
\hline \multicolumn{7}{|l|}{ Physical health } \\
\hline Health (self-estimated, 4-points) & $.164^{\dagger}$ & $.109^{*}$ & $.292^{\dagger}$ & $.372^{\dagger}$ & $.385^{\dagger}$ & $.443^{\dagger}$ \\
\hline \multicolumn{7}{|l|}{ Physical activity (high) } \\
\hline Average & $-.157^{*}$ & -.077 & $-.530^{\dagger}$ & -.030 & $-.312^{*}$ & -.265 \\
\hline Low & $-.314^{\dagger}$ & $-.306^{\dagger}$ & $-.526^{\dagger}$ & -.151 & $-.404^{*}$ & -.055 \\
\hline \multicolumn{7}{|l|}{ Vascular condition (none) } \\
\hline One & -.016 & .114 & -.074 & -.031 & .158 & -.056 \\
\hline Two or more & -.120 & -.011 & .013 & .073 & $.430^{*}$ & -.145 \\
\hline Diagnosed w/prostate disease & -.006 & & .055 & & .037 & \\
\hline Currently smoking & -.011 & $.153^{*}$ & .091 & .107 & -.113 & $.471^{*}$ \\
\hline \multicolumn{7}{|l|}{ Psychological health } \\
\hline Diagnosed w/depression & .173 & $.265^{\dagger}$ & $-.386^{\dagger}$ & -.127 & -.031 & -.443 \\
\hline Loss of job in past 3 years & .099 & .039 & -.009 & -.003 & .004 & .127 \\
\hline Divorced in past 3 years & $.453^{\dagger}$ & .060 & -.018 & .072 & .200 & -.020 \\
\hline Serious financial/work "setback" in past 3 years & $.205^{*}$ & -.146 & .001 & -.079 & .062 & -.058 \\
\hline Serious medical condition in past 3 years & -.025 & -.134 & .207 & -.172 & -.066 & .239 \\
\hline \multicolumn{7}{|l|}{ Sexual dysfunctions } \\
\hline Lacked interest in sex past year & $-.593^{\dagger}$ & $-.603^{\dagger}$ & $-.286^{\dagger}$ & $-.489^{\dagger}$ & $-.664^{\dagger}$ & $-.898^{\dagger}$ \\
\hline Experienced physical pain during sex past year & .100 & -.117 & $-.357^{*}$ & -.002 & .347 & $-.301^{*}$ \\
\hline Trouble achieving/maintaining erection past year & -.088 & & .084 & & .128 & \\
\hline Trouble becoming adequately lubricated past year & & -.152 & & .078 & & .175 \\
\hline \multicolumn{7}{|l|}{ Relationship characteristics and sexual practices } \\
\hline \multicolumn{7}{|l|}{ Marital status (married) } \\
\hline Cohabiting & -.153 & $.260^{*}$ & .139 & $.465^{*}$ & -.444 & .507 \\
\hline Dating & $.247^{*}$ & $.308^{*}$ & .043 & .002 & -.321 & -.051 \\
\hline \multicolumn{7}{|l|}{ Time horizon (together forever) } \\
\hline Hope to stay together & -.086 & $-.299^{\dagger}$ & .022 & -.015 & $.236^{*}$ & -.165 \\
\hline Less likely to stay together & .106 & $-.273^{*}$ & .028 & .043 & -.199 & -.298 \\
\hline Multiple sex partners & $.689^{\dagger}$ & $.495^{*}$ & $.213^{*}$ & $.424^{*}$ & -.080 & -.238 \\
\hline \multicolumn{7}{|l|}{ Frequency of sex (less than monthly) } \\
\hline Two or three times per month & $.619^{\dagger}$ & $.722^{\dagger}$ & $.543^{\dagger}$ & $.399^{\dagger}$ & $.853^{\dagger}$ & $.659^{\dagger}$ \\
\hline Weekly or more & $1.061^{\dagger}$ & $1.135^{\dagger}$ & $.719^{\dagger}$ & $.770^{\dagger}$ & $1.468^{\dagger}$ & $1.591^{\dagger}$ \\
\hline Foreplay time & $.118^{\dagger}$ & $.315^{\dagger}$ & $.280^{\dagger}$ & $.257^{\dagger}$ & $.385^{* *}$ & $.267^{\dagger}$ \\
\hline \multicolumn{7}{|l|}{ Real men sexual attitudes } \\
\hline Older people no longer want sex & $-.123^{\dagger}$ & -.053 & -.030 & $-.117^{\dagger}$ & $-.156^{\dagger}$ & $-.245^{\dagger}$ \\
\hline Men can enjoy sex even without love & $.059^{\dagger}$ & -.029 & .042 & -.051 & .032 & $-.141^{*}$ \\
\hline Real men' is ready for sex at any time & $.112^{* \dagger}$ & $.078^{\dagger}$ & $.224^{\dagger}$ & $.117^{\dagger}$ & $.142^{*}$ & $.149^{*}$ \\
\hline Women have greater control over sexual desires & .032 & $-.071^{\dagger}$ & .026 & -.000 & .016 & -.092 \\
\hline Women have a duty to meet partner's sexual needs & $.114^{\dagger}$ & .004 & $.140^{\dagger}$ & .046 & $.217^{\dagger}$ & $.239^{\dagger}$ \\
\hline \multicolumn{7}{|l|}{ Constant } \\
\hline$n$ & 4615 & 3913 & 3186 & 2688 & 1364 & 1078 \\
\hline$L^{2}$ & 578.57 & 665.19 & 541.49 & 515.72 & 331.12 & 386.95 \\
\hline
\end{tabular}

${ }^{*} p<.05$.

${ }^{\dagger} p<.01$. 
relationships. The corresponding figures for Clusters 2 and 3, comparing men with women, were $52 \%$ versus $41 \%$ and $30 \%$ versus $23 \%$, respectively. Differences of the percentage scores between men and women within each cluster were statistically significant at $p<.05$ for $t$-tests for all clusters and aspects of sexual well-being with one exception: there were no significant gender differences in Cluster 3 for physical and emotional satisfaction.

Second, the three clusters were largely ordered on the basis of the level of sexual well-being. Cluster 1 , which included most Western nations, manifested a high satisfaction pattern. Roughly two-thirds of men and women in this cluster reported that their relationships were pleasurable and emotionally satisfying and around $80 \%$ of them were satisfied with their current sexual functioning. However, only $49 \%$ of men and $32 \%$ of women indicated that sex was extremely or very important for their overall life.

In contrast, the other two clusters were quite distinct from Cluster 1. The second cluster exhibited moderate levels of satisfaction, with significantly lower percentages of positive assessments for emotional, physical, and sexual function satisfaction and a slightly larger gender gap in assessments of satisfaction regarding sexual function. However, both men and women in this cluster were statistically more likely to perceive sex as an important aspect of their overall life (men, 60\% women, 37\%) in comparison with Cluster 1. Countries in Cluster 3 tended to have populations that reported the lowest levels of emotional and physical satisfaction in their relationships, attributed even less importance to sex in their overall lives, and had a larger gender gap in satisfaction with sexual health. Overall, mean levels of emotional satisfaction, physical satisfaction, and satisfaction with sexual function appeared to be associated with one another across clusters, while the importance of sex was highest in Cluster 2, moderate in Cluster 1, and lowest in Cluster 3.

Figure 3 presents a scatterplot between satisfaction with sexual function and the average of two highly correlated indicators of emotional and physical satisfaction (i.e., relational satisfaction). This figure illustrates the clustered nature of subjective sexual well-being across the 29 countries. Both axes represent average percentage scores of men and women. Countries assigned to Cluster 1 are located in the upper right corner of Fig. 3, while those assigned to Cluster 2 are horizontally spread out in the center. This suggests higher levels of sexual wellbeing in Cluster 1 and a strong association between these two measures. In contrast, countries in Cluster 2 varied widely with respect to satisfaction with sexual function. Italy, Israel, Morocco, Turkey, and Brazil are located on the center and right side of the scatterplot, while the Asian countries are located on the left side. Countries assigned to Cluster 3 also showed a strong relationship between relational satisfaction and satisfaction with sexual function, but the scatterplot revealed lower levels of sexual well-being relative to the other clusters. This pattern suggests that it was the moderate level of relationship satisfaction reported in the countries assigned to Cluster 2 that distinguished them from the countries in Clusters 1 and 3 .

\section{Correlates of Subjective Sexual Well-Being}

We conducted exploratory factor analyses to assess the extent to which the four indicators reflected a unidimensional latent construct of sexual well-being. Results showed that the four aspects of subjective sexual wellbeing were not all tightly interrelated with one another (results available upon request). Factor analyses of the four aspects of subjective well-being, stratified by country and gender, showed the presence of a dominant factor in most countries both for men and women. Physical and emotional satisfaction loaded most strongly on this factor in all regions for both genders, while the importance of sex and sexual functional capacity were less strongly associated with this factor. Typically, the criterion for identified factors is having eigenvalues larger than 1 . Most countries showed eigenvalues around 2 for the first factor. The second factor, however, had eigenvalues close to 1 for most countries. ${ }^{12}$ Based on these results, we took a conservative, data-driven approach. We combined physical and emotional satisfaction for examining their predictors because separating them did not show much difference, but we separated them in predicting overall happiness since analyses revealed important differences between physical and emotional satisfaction.

Table II presents ordinary least squares regressions on relational satisfaction and ordered logistic regressions on satisfaction with sexual functioning and the importance of sex by gender and cluster. Predictors included demographic factors, measures for physical and psychological health, relationship characteristics, and sexual practices and attitudes. Regarding demographic covariates, age, an important proxy for overall health, was negatively

\footnotetext{
${ }^{12}$ We also inspected Cronbach's $\alpha$ values across countries and gender in order to assess internal reliability among the four aspects of sexual well-being. In most countries and both genders, the $\alpha$ values were between .60 and .80 , which just marginally support one underlying factor. Despite the striking sociocultural differences across the world regions, the fundamental similarity in the dominant factor across the three regions may attest to the presence of an underlying "universal" mechanism generating judgments about subjective sexual well-being.
} 
associated with indicators of sexual well-being, particularly with importance of sex, but was non-significant in many models controlling for other factors, including health status. In contrast, education and employment status did not show consistent patterns.

Several predictors of physical and psychological health showed strong, consistent patterns. Self-estimated health status positively and consistently affected all measures of sexual well-being across clusters and gender. The magnitudes of these effects were larger for Clusters 2 and 3 than Cluster 1, and tended to be stronger for women than men. These patterns may suggest less variation in Cluster 1 with respect to overall health as well as the dependent measures. The level of physical activity was positively associated with sexual well-being, but the pattern was gendered. Men who reported low physical activity had consistently lower levels of sexual wellbeing across clusters, while there appeared to be weaker associations for women.

With respect to psychological health, depression lowered relational satisfaction, but not satisfaction with sexual functioning or the importance of sex. Measures for sexual dysfunction, which reflect both psychological and physical problems, were strongly associated with sexual well-being. Lack of interest in sex lowered satisfaction with sexual function and the importance of sex for both men and women and across clusters, but only affected women's relational satisfaction. In contrast, the other types of sexual dysfunction, such as sexual pain, lubrication problems among women, and difficulty maintaining an erection among men primarily affected satisfaction with sexual functioning. These patterns of association between sexual dysfunction and subjective sexual well-being were largely consistent across the three clusters. Specific indicators of physical conditions (vascular conditions, prostate disease, serious medical conditions, and smoking status) or stressful life events (job loss, divorce, and financial setbacks) did not show significant, consistent patterns, controlling for general indicators of health and sexual dysfunctions.

Relationship characteristics and sexual practices also showed strong associations. An unexpected finding, contrary to prior research, was that nonmarital relationships, such as cohabitation and dating, were associated with higher levels of subjective sexual wellbeing than marriages, particularly for men. However, the level of commitment, as indexed by the time horizon, was positively associated with relational satisfaction and satisfaction with sexual functioning. Having multiple partners had a divergent pattern, particularly for men. Men who had multiple partners were less likely to be satisfied with their relationships, but more likely to believe that sex was important. The frequency of sex and the amount of time spent on foreplay also showed strong, positive associations for both men and women and across clusters with all the measures of sexual well-being.

Finally, some gender-related sexual attitudes appeared to exert the same pattern for men and women. Those who stereotypically thought that "real men" are ready for sex at any time or that women have a duty to meet their partner's sexual needs placed more importance on sex in their life. Men and women who thought men can have sex without love showed lower levels of relational satisfaction. Holding the belief that older people no longer want sex tended to reduce men's and women's evaluation of their satisfaction with sexual functioning and the assessment of the importance of sex.

\section{Sexual Well-Being and Overall Happiness}

We also investigated associations between aspects of subjective sexual well-being and respondents' assessments of their overall happiness. Table III provides the results of an ordered logit analysis; the log-odds coefficients calculated for each column can be compared across the clusters to evaluate the relative importance of a particular row variable. Again, only sexually active persons were included in the analysis because some of the independent variables (e.g., frequency of sex in the past year and time spent in foreplay) were not asked of sexually inactive persons.

The first two models of Table III, computed for the entire sample, showed robust effects for subjective sexual well-being. Controlling for all those significant factors related to health and life-course experiences, all four aspects of sexual well-being were significantly related to overall happiness in the second model. Among the four aspects, physical and emotional satisfaction with partner had stronger effects than the other two aspects. For example, in Model 2, the log-odds for physical and emotional satisfaction with partner were $.640(p<.001)$ and .508 ( $p<.001)$, respectively. In contrast, satisfaction with sexual health was only $.114(p<.001)$ and the importance of sex only $.136(p<.001)$. More interestingly, older individuals and women showed higher overall wellbeing once we controlled for sexual well-being, indicating that lower sexual well-being among women and older adults suppressed their overall happiness. In addition, the inclusion of sexual well-being in Model 2 also reduced the positive effects of health and physical activity on general happiness.

The next three models were computed for each cluster to examine possible variations in the effects 
Table III. Ordered Logit of Overall Happiness by Cluster

\begin{tabular}{|c|c|c|c|c|c|}
\hline Variables & Total sam & & Cluster 1 & Cluster 2 & Cluster 3 \\
\hline \multicolumn{6}{|l|}{ Control variables } \\
\hline Age & $.006^{\dagger}$ & $.021^{\dagger}$ & $.018^{\dagger}$ & $.018^{\dagger}$ & $.032^{\dagger}$ \\
\hline Women & -.027 & $.150^{\dagger}$ & .069 & .479 & -.031 \\
\hline \multicolumn{6}{|l|}{ Education (high school) } \\
\hline Less than high school & -.055 & $-.081^{*}$ & -.092 & $-.123^{*}$ & -.013 \\
\hline Higher than high school & $.089^{\dagger}$ & .061 & .069 & .022 & .038 \\
\hline \multicolumn{6}{|l|}{ Employment status (working) } \\
\hline Homemaker & -.019 & .062 & .049 & .138 & .090 \\
\hline Retired & $.127^{\dagger}$ & .070 & $.123^{*}$ & -.034 & .109 \\
\hline Unemployed & $-.307^{\dagger}$ & $-.297^{\dagger}$ & $-.211^{*}$ & $-.341^{\dagger}$ & $-.414^{*}$ \\
\hline Health (self-estimated, 4-points) & $.822^{\dagger}$ & $.582^{\dagger}$ & $.587^{\dagger}$ & $.490^{\dagger}$ & $.808^{\dagger}$ \\
\hline \multicolumn{6}{|l|}{ Physical activity (high) } \\
\hline Average & $-.330^{\dagger}$ & $-.245^{\dagger}$ & $-.207^{\dagger}$ & $-.229^{\dagger}$ & $-.418^{\dagger}$ \\
\hline Low & $-.586^{\dagger}$ & $-.470^{\dagger}$ & $-.460^{\dagger}$ & $-.455^{\dagger}$ & $-.620^{\dagger}$ \\
\hline Currently smoking & $-.090^{\dagger}$ & $-.073^{*}$ & -.014 & $-.148^{\dagger}$ & .053 \\
\hline Diagnosed w/depression & $-.373^{\dagger}$ & $-.361^{\dagger}$ & $-.391^{\dagger}$ & $-.385^{\dagger}$ & .273 \\
\hline Loss of job in past 3 years & $-.208^{\dagger}$ & $-.199^{\dagger}$ & $-.329^{\dagger}$ & -.137 & .025 \\
\hline Divorced in past 3 years & $-.188^{*}$ & $-.307^{\dagger}$ & $-.294^{*}$ & $-.283^{*}$ & -.247 \\
\hline Serious financial/work "setback" in past 3 years & $-.481^{\dagger}$ & $-.367^{\dagger}$ & $-.514^{\dagger}$ & $-.227^{\dagger}$ & $-.301^{\dagger}$ \\
\hline \multicolumn{6}{|l|}{ Marital status (married) } \\
\hline Cohabiting & .076 & -.097 & $-.152^{*}$ & -.230 & .013 \\
\hline Dating & $-.171^{\dagger}$ & $-.424^{\dagger}$ & $-.499^{\dagger}$ & $-.400^{\dagger}$ & -.097 \\
\hline \multicolumn{6}{|l|}{ Sexual well-being factors (5-points) } \\
\hline Physical pleasure with partner & & $.640^{\dagger}$ & $.458^{\dagger}$ & $.928^{\dagger}$ & $.515^{\dagger}$ \\
\hline Emotional pleasure with partner & & $.508^{\dagger}$ & $.575^{\dagger}$ & $.394^{\dagger}$ & $.763^{\dagger}$ \\
\hline Satisfaction with sexual function/health & & $.081^{\dagger}$ & $.114^{\dagger}$ & $-.090^{*}$ & .073 \\
\hline Importance of sex in life & & $.098^{\dagger}$ & $.136^{\dagger}$ & $.124^{\dagger}$ & .053 \\
\hline \multicolumn{6}{|l|}{ Sexual well-being by gender } \\
\hline Physical pleasure $\times$ women & & & .087 & -.113 & $-.421^{\dagger}$ \\
\hline Emotional pleasure $\times$ women & & & $-.124^{*}$ & -.059 & $.437^{\dagger}$ \\
\hline Satisfaction with Function $\times$ women & & & .078 & .074 & -.035 \\
\hline Importance of sex $\times$ women & & & -.041 & -.042 & .159 \\
\hline$n$ & 20491 & 20438 & 10406 & 6934 & 3098 \\
\hline$L^{2}$ & 3584.5 & 8342.2 & 3705.3 & 2735.0 & 1297.4 \\
\hline
\end{tabular}

of sexual well-being on happiness across clusters and gender. Compared with satisfaction with sexual function or importance of sex, physical and emotional satisfaction showed stronger positive effects on overall happiness across clusters and gender. There appeared to be larger effects for Clusters 2 and 3 with respect to physical (for Cluster 2) and emotional satisfaction (for Cluster 3) than for Cluster 1. This was true both for men (main effects of sexual well-being) and women (the sum of main and interaction effects). Note the paradox here: although Clusters 2 and 3 generally had lower levels of positive evaluation of their subjective sexual well-being when compared with Cluster 1, the association between sexual well-being and happiness was stronger in these clusters than in Cluster 1. In contrast, the positive role of satisfaction with sexual function for general happiness was most distinctive for Cluster 1. Its role was mildly negative for men $(-.09)$ or nonexistent for women $(-.09$ $+.07=-.02$ ) for Cluster 2, and similarly weak for Cluster 3. Lastly, the patterns of interactions with gender revealed asymmetries across clusters. The role of physical satisfaction in predicting overall happiness for women compared with men became weaker while the role of emotional satisfaction for women, by contrast, became stronger across clusters (that is, note the changes in the coefficients for the two interactions as one proceeds from Cluster 1 to Cluster 3). The overall happiness of women aged 40-80 years in a male-centered sexual regime with the lowest average subjective sexual wellbeing (i.e., Cluster 3) was much more affected by their emotional satisfaction with their sexual partners than by their physical satisfaction, compared with their male 
counterparts. All four aspects of subjective sexual wellbeing were found to be significantly related to overall happiness.

\section{DISCUSSION}

There was evidence of a strongly gendered response to subjective sexual well-being, where men reported higher levels of satisfaction than women, regardless of sociocultural context. The results of this study showed that mean levels of satisfaction were, in general, lower for women than men across all three clusters for all four aspects of subjective sexual well-being. In one sense, this finding was surprising, since we expected that gender would not be relevant in the gender-equal cluster. However, we did find that gender differences were greater in the male-centered clusters. This pattern suggests that the type of gender regime is important for gender differences in sexual well-being, but true parity remains an ideal even in countries where beliefs about gender equality are more widespread. We also note that this finding about gendered differences in sexual wellbeing was in sharp contrast to the common finding that women report experiencing greater happiness and more intense positive emotions than do men (Nolen-Hoeksema \& Rusting, 1999).

There was also evidence of substantial cross-national clustering in the level of sexual well-being. The three clusters were highly ordered: countries in Cluster 1 exhibited the highest levels of sexual satisfaction with their partners, and both women and men were quite satisfied with their sexual health. Cluster 2 , in contrast, had moderate levels of relationship satisfaction, but attributed more importance to sex than Cluster 1. Cluster 3 had the lowest levels of sexual well-being for all indicators. These results support the notion that the ideal of companionate relationships tends to value positively sexual competencies, interests, and performance between intimate sex partners. In other words, sex in companionate relationships serves not only reproductive purposes, but also expresses the quality of the relationship. Male-centered regimes, where sexual behavior is more oriented toward reproduction, tend to discount the relational meaning of sex and the importance of sexual pleasure for women.

There were also important differences between the two male-centered regimes. Cluster 2 appeared to be a hybrid of the two other clusters. Figure 3 showed that Cluster 2 countries were located in the middle on the axis for relationship satisfaction, but were spread out with respect to the axis for satisfaction with sexual function. Countries in Cluster 2 were also internally diverse compared with those of the other two clusters. Why sex would be more important in Cluster 2 than Cluster 1 remains an interesting, unexpected finding. One possible explanation for this pattern was that although pleasing one's sexual partner was an important value of relationships in the gender-equal regime, sex was not the only basis of the relationship. In contrast, in male-centered regimes, sex was more likely to be thought of as a duty that was fundamental to the intimate relationship. The association between the importance of sex and gendered attitude about sex as a duty was revealed in Table II, where belief in "real men" or women's duty showed positive effects on the importance of sex. In any case, it is clear that countries in Cluster 2, relative to countries in Cluster 1, produced less relationally satisfying relationships.

The consistently low levels of sexual well-being in many Asian countries was quite interesting. A prior study on cross-national variation in sexual attitudes found that the two Asian countries included in the analysis, Japan and the Philippines, were outliers in comparison with the sexual regimes of Western nations (Widmer et al., 1998). In our study, we found that the Asian countries all reported low levels of satisfaction with sexual function and moderate to low levels of satisfaction with their relationships and the importance of sex. Given the substantial, observed differences in sexual attitudes, practices, and sexual well-being between "East" and "West," we suggest that future research could fruitfully investigate cultural and structural causes of this variation.

Correlates of subjective sexual well-being showed both consistency and independence across aspects of sexual well-being and gender. Self-rated health and sexual practices, such as frequency of sex or extent of foreplay, were strong, consistent correlates of subjective sexual well-being across the clusters and gender. Independence among the different aspects of subjective sexual wellbeing was also evident. For example, depression showed negative associations with relational satisfaction only and having multiple partners was negatively associated with relational satisfaction but had a positive association with the importance of sex in one's life. Several biological factors manifested gendered patterns: negative effects of age, lower physical activity, and smoking were more apparent for men than for women. Smoking clearly has implications beyond strict biological effects because women who smoked in male-centered sexual regimes enjoyed even higher satisfaction with sexual function and emphasized the importance of sex in one's life more than women in the gender-equal regime. 
Differences among clusters were more distinctive in how subjective sexual well-being affected overall happiness, rather than in which factors were predictors of subjective well-being. Both physical and emotional satisfaction were strong predictors of overall happiness for all clusters. However, women's inclinations to weigh emotional satisfaction more while downplaying physical satisfaction in assessing overall happiness were strikingly salient in the Cluster 3 male-centered, low satisfaction regime.

There were several limitations to this study. First, the GSSAB incorporated multiple modes for administering the surveys, each with different sampling methods (Laumann et al., 2005; Nicolosi et al., 2004). This limits the generalizability of our findings, since the intercept and door-to-door protocol were not representative of national populations. It is also possible that mode of administration affected how people answered survey questions. However, previous studies have shown that the difference between in-person interviews and selfcompleted or mailed questionnaires does not affect the estimates of the prevalence of sexual behavior (McEwan, Harrington, Bhopal, Madhok, \& McCallum, 1992; Nebot et al., 1994; Wadsworth, Field, Johnson, Bradshaw, \& Wellings, 1993).

Second, the overall response rate was not high, but was greater in the countries in which the offer of participation involved personal contact. People were reluctant to refuse a personal contact, but were more at ease in declining if the request was made by an anonymous caller at the other end of a telephone line (Weinhardt, Forsyth, Carey, Jaworski, \& Durant, 1998). The low response rates may create sample selection bias by including individuals who are either more interested in the topic or who have more time to answer questions. In our case, we believe that the latter was more important given the policy of not trying to convert initial refusals. There was no reason to think that availability to answer survey questions was related to any of the variables included in the analysis. Nevertheless, the former issue may still be relevant; thus, the GSSAB may tend to include individuals who were more interested in discussing sexual issues.

To check for possible biases related to mode of administration and low response rates, we compared GSSAB estimates of sexual problems, sexual satisfaction, and general happiness with published results. Wherever possible, the country-specific results produced by the GSSAB surveys have been compared with those produced by other published studies, and the estimates of prevalence of sexual problems were often similar to or lower than the values reported in the other studies. Nevertheless, it is difficult to compare our results with those of previous studies because few of them were based on population samples, many included individuals younger than 40 years old, and their definitions of sexual problems, sexual wellbeing, and happiness were often different. However, our findings were consistent with those previous population studies of the prevalence of erectile dysfunction (Blanker et al., 2001; Nicolosi et al., 2003; Prins, Blanker, Bohnen, Thomas, \& Bosch, 2002), even if our estimates were slightly lower, because we used a more specific screening method. The prevalence of early ejaculation in men (Blanker et al., 2001; Carson, Glasser, Laumann, West, \& Rosen, 2003; Helgason et al., 1996; Laumann et al., 1999; Prins et al., 2002) and other sexual dysfunctions in women (Anastasiadis, Davis, Ghafar, Burchardt, \& Shabsigh, 2002; Berman, Berman, \& Goldstein, 1999; Dunn, Croft, \& Hackett, 1999; Fugl-Meyer \& FuglMeyer, 1999; Kadri, McHichi Alami, \& McHakra Tahiri, 2002; Laumann et al., 1999; Richters, Grulich, de Visser, Smith, \& Rissel, 2003; Simons \& Carey, 2001) reported in the few other population studies carried out in various parts of the world were generally consistent with that of the region-specific prevalence found by us (Laumann et al., 2005; Nicolosi et al., 2004). While there are only a handful of studies investigating subjective sexual well-being with comparable questions (Cain et al., 2003; Haavio-Mannila \& Kontula, 1997; Laumann et al., 1994; Oberg et al., 2002), there are more nationally based studies of happiness (Diener \& Suh, 1999) and we found those estimates to be generally consistent with those we observed.

Third, the exclusion of sexually inactive individuals in many analyses may further limit the generalizability of our findings by creating sample selection effects. Since there was likely to be an association between poor sexual well-being and sexual inactivity, we expected that effects of some predictors, such as age, physical health, and psychological well-being, were actually underestimated. Thus, we view this study as a conservative test of the correlates of sexual well-being.

The concept of happiness or subjective well-being has been discussed extensively in the psychological, psychoanalytic, and psychiatric literature. The website entitled the World Database of Happiness is "an ongoing register of scientific research on subjective appreciation of life" (World Database of Happiness). However, although there are a handful of country-specific studies of selected aspects of subjective sexual well-being (Laumann et al., 1994; Oberg et al., 2002), comparable cross-country studies of subjective sexual well-being, as defined here, do not exist. 
In sum, we have investigated four aspects of sexual well-being - emotional and physical satisfaction with partnered sexual relationship, satisfaction with sexual health/function, and the importance of sex as a part of life as a whole. From our findings, we have drawn the following conclusions: (1) despite substantial cultural variation, several predictors of subjective sexual wellbeing (physical and mental health, sexual practices, and relationship context) were consistent across world regions; (2) there was strong evidence for a gendered response to subjective sexual well-being, regardless of sociocultural context; and (3) subjective sexual well-being was correlated with overall happiness in both men and women.

\section{ACKNOWLEDGMENTS}

The Global Study of Sexual Attitudes and Behaviors (GSSAB) was funded by Pfizer Inc. As independent consultants charged with directing the design, analysis, and interpretation of the collected data, a scientific advisory board was assembled to include researchers and practitioners whose primary institutional affiliations are with universities or government research institutes or in public or private practice. The analysis and interpretation of the results are the sole product of discussions among the co-authors, represent the consensus of the co-authors, and have not been subject to editorial revision by Pfizer Inc. The authors acknowledge the contribution of their colleagues on the international advisory board for this study: Gerald Brock (Canada), Jacques Buvat (France), Uwe Hartmann (Germany), Sae-Chul Kim (Korea), Rosie King (Australia), Ken Marumo (Japan), Ferruh Simsek (Turkey).

\section{REFERENCES}

Anastasiadis, A. G., Davis, A. R., Ghafar, M. A., Burchardt, M., \& Shabsigh, R. (2002). The epidemiology and definition of female sexual disorders. World Journal of Urology, 20, 74-78.

Berman, J. R., Berman, L., \& Goldstein, I. (1999). Female sexual dysfunction: Incidence, pathophysiology, evaluation, and treatment options. Urology, 54, 385-391.

Blanker, M. H., Bosch, J. L. H. R., Groeneveld, F. P. M. J., Bohnen, A. M., Prins, A., Thomas, S., et al. (2001). Erectile and ejaculatory dysfunction in a community-based sample of men 50-78 years old: Prevalence, concern, and relation to sexual activity. Urology, 57, 763-768

Blumstein, P., \& Schwartz, P. (1983). American couples: Money, work, sex. New York: Morrow.

Braun, M., Wassmer, G., Klotz, T., Reifenrath, B., Mathers, M., \& Engelmann, U. (2000). Epidemiology of erectile dysfunction: Results of the "Cologne Male Survey." International Journal of Impotence Research, 12, 305-311.

Cain, V. S., Johannes, C. B., Avis, N. E., Mohr, B., Schocken, M., Skurnick, J., et al. (2003). Sexual functioning and practices in a multi-ethnic study of midlife women: Baseline results from SWAN. Journal of Sex Research, 40, 266-277.

Carson, C. C., Glasser, D. B., Laumann, E. O., West, S. L., \& Rosen, R. C. (2003). Prevalence and correlates of premature ejaculation among men aged 40 years and older: A United States nationwide population-based study [Abstract 1249]. Journal of Urology, 169, 321.

Diener, E., \& Suh, E. (1999). National differences in subjective wellbeing. In D. Kahneman, E. Diener, \& N. Schwartz (Eds.), Wellbeing: The foundations of hedonic psychology (pp. 434-450). New York: Sage.

Dunn, K. M., Croft, P. R., \& Hackett, G. I. (1999). Association of sexual problems with social, psychological, and physical problems in men and women: A cross-sectional population survey. Journal of Epidemiology and Community Health, 53, 144-148.

Feldman, H. A., Goldstein, I., Hatzichristou, D. G., Krane, R. J., \& McKinlay, J. B. (1994). Impotence and its medical and psychosocial correlates: Results of the Massachusetts Male Aging Study. Journal of Urology, 151, 54-61.

Fugl-Meyer, A. R., \& Fugl-Meyer, K. S. (1999). Sexual disabilities, problems and satisfaction in 18-74-year-old Swedes. Scandinavian Journal of Sexology, 2, 79-105.

Haavio-Mannila, E., \& Kontula, O. (1997) Correlates of increased sexual satisfaction. Archives of Sexual Behavior, 26, 399-419.

Helgason, A. R., Adolfsson, J., Dickman, P., Arver, S., Fredrikson, M., Gothberg, M., et al. (1996). Sexual desire, erection, orgasm and ejaculatory functions and their importance to elderly Swedish men: A population-based study. Age Ageing, 25, 285-291.

Kadri, N., McHichi Alami, K. H., \& McHakra Tahiri, S. (2002). Sexual dysfunction in women: Population-based epidemiological study. Archives of Women's Mental Health, 5, 59-63.

Kahneman, D., Diener, E., \& Schwartz, N. (Eds.). (1999). Well-being The foundations of hedonic psychology. New York: Russell Sage Foundation.

Laumann, E. O., Gagnon, J., Michael, R. T., \& Michaels, S. (1994). The social organization of sexuality: Sexual practices in the United States. Chicago: University of Chicago Press.

Laumann, E. O., Nicolosi, A., Glasser, D. B., Paik, A., Gingell, C., Moreira, E., et al. (2005). Sexual problems among women and men aged 40-80 years: Prevalence and correlates identified in the Global Study of Sexual Attitudes and Behaviors. International Journal of Impotence Research, 17, 39-57.

Laumann, E. O., Paik, A., \& Rosen, R. C. (1999). Sexual dysfunction in the United States: Prevalence and predictors. JAMA, 281, 537544.

McEwan, R. T., Harrington, B. E., Bhopal, R. S., Madhok, R., \& McCallum, A. (1992). Social surveys in HIV/AIDS: Telling or writing? A comparison of interview and postal methods. Health Education Research, 7, 195-202.

Moreira, E. D. Jr., Abdo, C. H. N., Torres, E. B., Lobo, C. F. L., \& Fittipaldi, J. A. S. (2001). Prevalence and correlates of erectile dysfunction: Results of the Brazilian Study of Sexual Behaviour. Urology, 58, 583-588.

Nebot, M., Celentano, D. D., Burwell, L., Davis, A., Davis, M., Polacsek, M., et al. (1994). AIDS and behavioural risk factors in women in inner city Baltimore: A comparison of telephone and face-to-face surveys. Journal of Epidemiology and Community Health, 48, $412-418$.

Nicolosi, A., Laumann, E. O., Glasser, D. B., Moreira, E., Paik, A., \& Gingell, C. (2004). Sexual behavior and sexual dysfunctions after age 40: The Global Study of Sexual Attitudes and Behaviors. Urology, 64, 991-997.

Nicolosi, A., Moreira, E. D., Shirai, M., Bin Mohd Tambi, M. I. \& Glasser, D. B. (2003). Epidemiology of erectile dysfunction in four countries: Cross-national study of the prevalence and correlates of erectile dysfunction. Urology, 61, 201206.

Nolen-Hoeksema, S., \& Rusting, C. (1999). Gender differences in well-being. In D. Kahneman, E. Diener, \& N. Schwarz (Eds.), 
Well-being. The foundations of hedonic psychology (pp. 330-350). New York: Russell Sage Foundation.

Oberg, K., Fugl-Meyer, K. S., \& Fugl-Meyer, A. R. (2002). On sexual well-being in sexually abused Swedish women: Epidemiological aspects. Sexual and Relationship Therapy, 17, 329341 .

Prins, J., Blanker, M. H., Bohnen, A. M., Thomas, S., \& Bosch, J. L. (2002). Prevalence of erectile dysfunction: A systematic review of population-based studies. International Journal of Impotence Research, 14, 422-432.

Reiss, I. L. (1967). The social context of premarital sexual permissiveness. New York: Holt, Rinehart, \& Winston.

Reiss, I. L., \& Reiss, H. M. (1997). Solving America's sexual crises. Amherst, NY: Prometheus.

Richters, J., Grulich, A. E., de Visser, R. O., Smith, A. M., \& Rissel, C. E. (2003). Sexual difficulties in a representative sample of adults. Australia New Zealand Journal of Public Health, 27, 164170.

Schwartz, N., \& Strack, F. (1999). Reports of subjective wellbeing: Judgmental processes and their methodological implications. In D. Kahneman, E. Diener, \& N. Schwartz (Eds.), Well-being: The foundations of hedonic psychology (pp. 61-84). New York: Russell Sage Foundation.
Simons, J. S., \& Carey, M. P. (2001). Prevalence of sexual dysfunctions: Results from a decade of research. Archives of Sexual Behavior, $30,177-219$.

Wadsworth, J., Field, J., Johnson, A. M., Bradshaw, S., \& Wellings, K. (1993). Methodology of the National Survey of Sexual Attitudes and Lifestyles. Journal of the Royal Statistical Society Series A, $156,407-421$.

Waite, L. J., \& Joyner, K. (2001). Emotional and physical satisfaction with sex in married, cohabiting, and dating sexual unions: Do men and women differ? In E. O. Laumann \& R. T. Michael (Eds.), Sex, love, and health in America: Private choices and public policies (pp. 239-269). Chicago: University of Chicago Press.

Ward, J. H. (1963). Hierarchical grouping to optimize an objective function. Journal of the American Statistical Association, 58, 236244.

Weinhardt, L. S., Forsyth, A. D., Carey, M. P., Jaworski, B. C., \& Durant, L. E. (1998). Reliability and validity of self-report measures of HIV-related sexual behavior: Progress since 1990 and recommendations for research and practice. Archives of Sexual Behavior, 27, 155-180.

Widmer, E. D., Treas, J., \& Newcomb, R. (1998). Attitudes toward nonmarital sex in 24 countries. Journal of Sex Research, 35, 349-358.

World Database of Happiness. Available from URL: http:/ /www.eur.nl/ $\mathrm{fsw} / \mathrm{research} /$ happiness. 